\title{
Dynamical behaviors of the stochastic multimolecule oscillatory reaction model with Poisson jumps
}

\author{
Yongchang $\mathrm{Wei}^{1}$ and Zongbin $\mathrm{Yin}^{2}$ \\ ${ }^{1}$ Yangtze University \\ ${ }^{2}$ Guangzhou College of Technology and Business
}

November 10, 2020

\begin{abstract}
This paper reveals long time dynamics in the stochastic multimolecule oscillatory reaction model with Poisson jumps. First, this system is proved to have a unique global positive solution via the Lyapunov technique. Second, the existence and uniqueness of general random attractors for its stochastic homeomorphism flow is proved by the comparison theorem, and meanwhile, a criterion for the existence of singleton sets is obtained. Finally, numerical simulations are used to illustrate the predicted random attractors.
\end{abstract}

\section{Hosted file}

dynamic-MMA.pdf available at https://authorea.com/users/374523/articles/492059-dynamicalbehaviors-of-the-stochastic-multimolecule-oscillatory-reaction-model-with-poisson-jumps 